\title{
B STAR COLOURS BETWEEN 2000 AND 3000 ANGSTROMS
}

\author{
by Albert Bogarss, III
}

(Goddard Space Flight Center

(National Aeronautics and Space Administration Greenbelt, Maryland, U. S. A.)

RÉsumé. _- On a déterminé au moyen de photomètres à filtres placés à bord de fusées, les flux ultraviolets (d 2200 et $2600 \AA$ ) de 180 étoiles $A$ et $B .48$ de ces étoiles ont été mesurées plus d'une fois et l'analyse des données indique une incertitude inférieure à $20 \%$. En traçant le diagramme indice de couleur $U$-V en fonction de $B$ - $V$ pour des étoiles de classes de luminosité $I I I, I V$ et $V$ et de faible rougissement, on trouve un taux de corrélation compatible avec les erreurs. Les résultats semblent être d'une qualité suffisante pour qu'on en déduise les températures des étoiles $B$ et les courbes de diffusion interstellaire.

ABSTRACT. - Ultraviolet fluxes at 2200 and $2600 \AA$ have been measured for about $180 \mathrm{~B}$ and A stars using filter photometers in rockets. Forty-eight of these stars have been observed more than once, and an analysis of these data show the uncertainty of a single observation to be less than $20 \%$. A U-V color versus B-V plot of luminosity class III, $I V$ and $V$ stars with little reddening shows a degree of correlation consistent with the observational uncertainty. The data appears to be of sufficient quality for use in deriving B star temperatures and interstellar scattering curves.

Резюме. - При посредстве форотометров с фильтрами, помещенных на борту ракет, были определены ультрафиолетовые потоки (от 2200 до 2600 А) 180 звезд А и В. 48 из этих звезд были измерены неоднократно и анализ данных указывает на недостоверность меньше 20 \%. Построив диаграмму показателяцвета U-V в зависимости от В-V для звезд классов светимости III, IV и V и со слабым покраснением, найден корреляционный коэффициент совместимый с опибками. Результаты, как будто, качественно достаточны, чтобы из них вывеста температуры звезд В и кривые межзвездного рассеяния.

Ultraviolet astronomical observations have suffered due to limitations in detector systems, limitations in photometric standards, the problem of obtaining precision data with miniature instrumentation in the rocket environment, and the inability to select the stars to be observed. It is these restrictions which have dictated the kinds of cbservations that have been made. The first program to be undertaken was broadband ultraviolet colour and flux measurements using small, simple photometers that scanned the sky with the rocket motion. This data is of value in its own right and also has been particularly useful in the design of more sophisticated experiments. As instrumentation techniques improved, it became possible to fly medium resolution objective grating spectrometers to record ultraviolet flux distributions. This data is discussed in the following paper. Now that rocket pointing systems are becoming available, a relatively high resolution scanning spectrometer is being designed to study selected stars in greater detail.

Perhaps the two greatest difficulties associated with the work done to date are that the rockets could not be pointed and thet the equipment is destroyed after each flight. Because of the inability to point the rockets, an essentially random selection of stars is obtained on any given flight, and it is necessary to combine the data from a number of flights to produce a useful set of observations. But here the second difficulty is encountered. Since the instruments are destroyed at the end of flight, the internal consistency of the results is totally dependent upon the maintenonce of laboratory photometric standards which can be used to calibrate each set of rocket instrumentation and to transfer the calibration from one flight to the next. Because of the emphasis that has been placed on these standards, and because they can be maintained only by repeated absolute calibration, the uncertainties in the absolute calibration of the data are generally smaller than the random uncertainties within the observations themselves. Only by paying careful attention to these calibrations and by demonstrating their consistency from one flight to the next can it be argued that the data is worth interpreting. My principal aim here is to state what kind of accuracy has been achieved. 
In obtaining the filter photometry data, two wavelengths have been used : 2600 and $2200 \AA$; and the photometers have been flown in pairs : one with a $2600 \AA$ filter and one with a $2200 \AA$ filter. The photometers in a pair have parallel optical axes so that simultaneous flux measurements are made at both wavelengths. Originally $60 \mathrm{~mm}$ diameter quartz or calcium fluoride lenses were used as objectives; more recently $150 \mathrm{~mm}$ diameter Cassegrain telescopes have been employed as collectors. In either case, the filter is placed behind the collector, a field stop and field lens are placed at the focal plane, and a quartz windowed photomultiplier is used as a detector. The filter characteristics are shown in Figures 1 and 2.

The first filter has an effective wavelength of $2200 \AA$ with a $210 \AA$ effective width. It is a first order interference filter and exhibits a long wavelength tail which contributes significantly to the transmitted energy. On the short wavelength side the tail is chopped by the transmission of the optical system. Since the long wavelength tail extends into the bandpass of the $2600 \AA$ filter, its effect on the $2200 \AA$ flux can be allowed for by subtracting a fixed percentage of the flux seen by the $2600 \AA$ filter. This procedure is accurate to within a percent or so for a wide range of spectral gradients. The filter shown in Figure 2 is a sandwich of lead doped potassium bromide-potas- sium chloride and nickel sulphate hexahydrate crystals together with various high silica glasses. It has an effective wavelength of $2600 \AA$ with a $250 \AA$ bandpass. The calibration of the photometers is fairly straightforward. The transmission of the optical train is measured with a spectrophotometer, and the relative spectral efficiency of the photomultiplier is compared against a known phosphor. Since both filters pass the $2537 \AA$ line of mercury, this line can be used conveniently as an absolute standard in an inverse square law calibration. The mercury standard is maintained by periodically using a thermocouple to compare it to the total radiation from a tungsten lamp calibrated by the National Bureau of Standards.

Three pairs of photometers are flown in a rocket, each pair looking in a different direction in order to cover as much sky as possible. As the rocket spins and precesses, the photometers scan the sky, typically seeing an individual star for about twotenths of a second. In four flights some 300 two colour observations on about 180 different stars have been recorded. Forty-eight of these stars have been observed with more than one pair of photometers. Therefore, there are approximately 120 observations, covering forty-eight stars, from which the internal reliability of the data can be judged. Of these forty-eight stars, all observations clustered within $20 \%$ for forty of them,

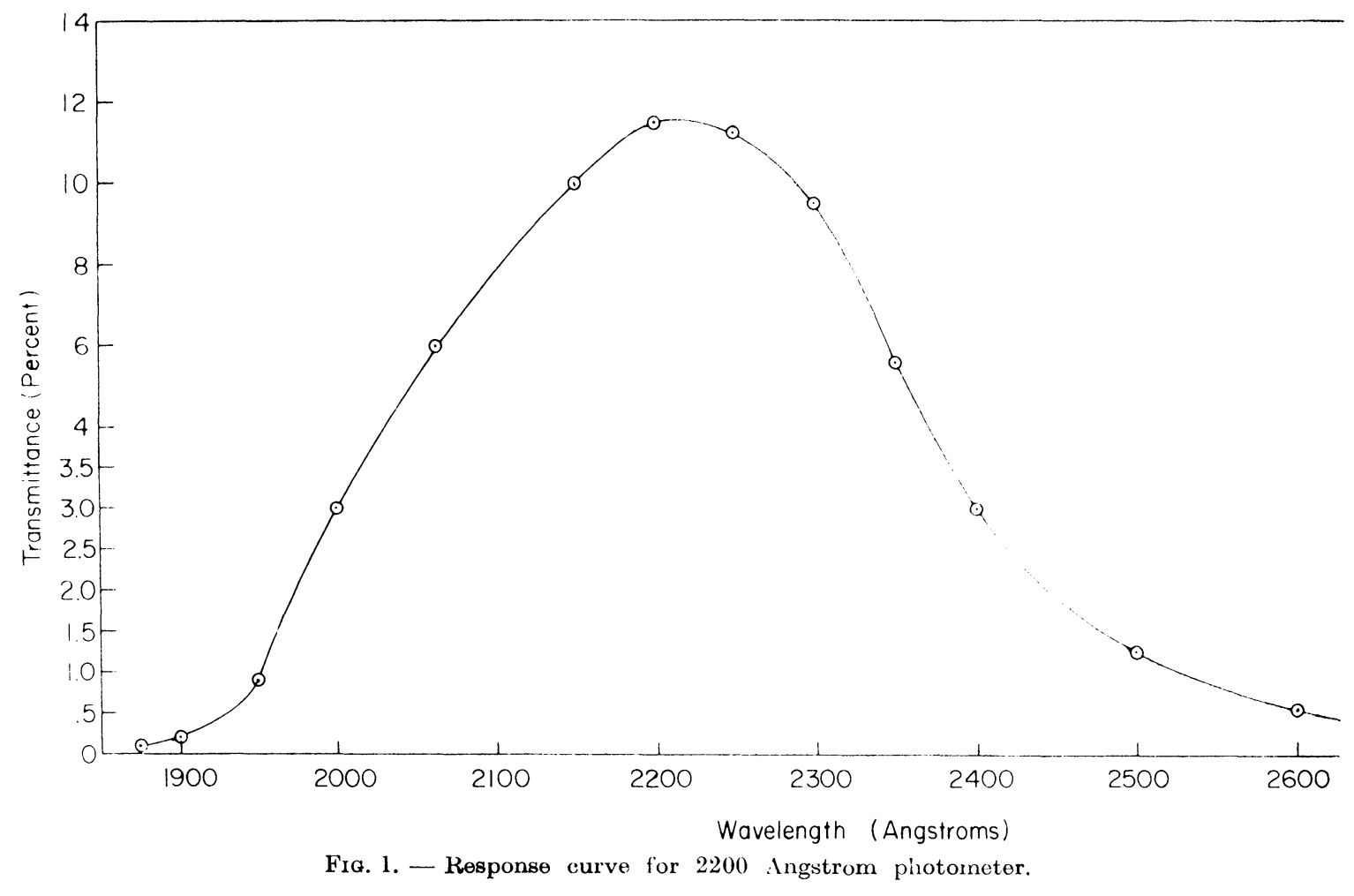




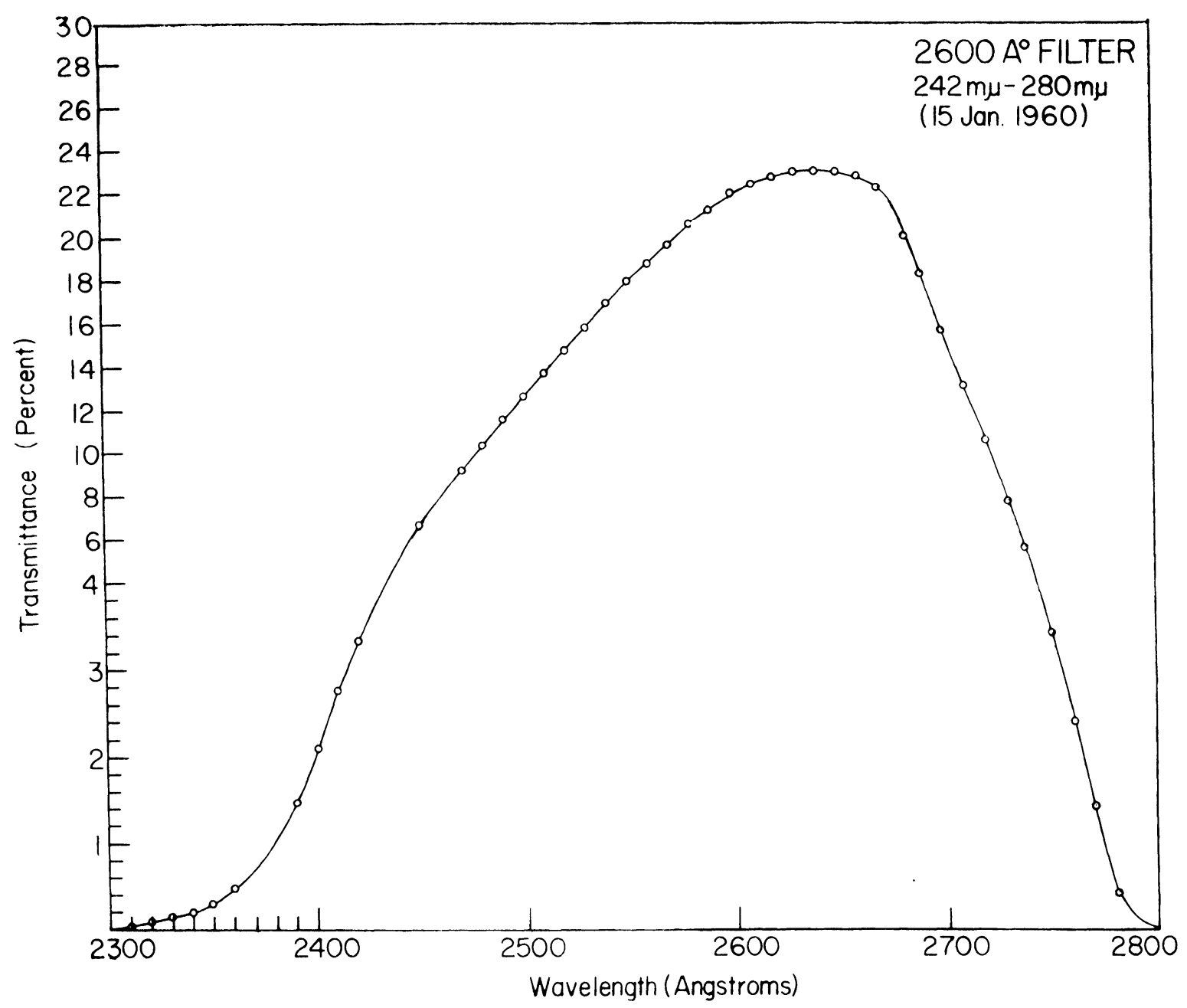

Fig. 2. - Response curve for 2600 Angstrom photometer.

within $10 \%$ for seventeen. The uncertainties in this data should be representative of the accuracy of all the observations, so that even for those stars observed cnly once the data should have an uncertainty of less than $20 \%$

It is interesting to see the colours derived for the 48 well observed stars, and to compare them with conventional $\mathrm{B}-\mathrm{V}$ colours. Here again, one is subjected to a severe selection process. When the selection is restricted to those whose $B-V$ is available, to luminosity classes III, IV, and V, and to those with colour excess of .02 magnitude or less, only thirteen of the original forty-eight stars are left, and they are shown in Table I and Figure 3. $\beta$ Per and $\beta$ (ep are vaciable, but the variability in $(B-V)$ is small compared to the uncertainty in the ultraviolet colours. $\varepsilon$ UMa is a peculiar star showing chromium and europium lines ; however, with such a small group of stars to work with,
TABLE I

UITRAVIOLET COIOURS of Stiars with tittite Colour Excess.

\begin{tabular}{lllcc}
\hline & & Sp & B-V & $m_{2200}-m_{2600}$ \\
$\zeta$ & - & - & - & -.06 \\
$\gamma$ & UMa & A2 V & +.02 & -.00 \\
$\alpha$ & UMa & A0 V & .00 & .00 \\
$\Sigma$ & CrB & A0 V & -.02 & -.14 \\
$\beta$ & UMa & A0 V & -.03 & -.08 \\
$\beta$ & Per & B8 & -.07 & -.32 \\
$\zeta$ & Lib & B8 V & -.11 & -.35 \\
$\tau$ & Dra & B6 III & -.12 & -.45 \\
$\zeta$ & Her & B5 IV & -.15 & -.66 \\
$\eta$ & Her & B3 V & -.18 & -.53 \\
$\mu_{1.2}$ & Sco & B3 V & -.20 & -.37 \\
3 & Bep & B2 III & -.20 & -.36 \\
$\eta$ & Lup & B2 V & -.22 & -.49 \\
$\eta$ & & & & -.55 \\
\hline
\end{tabular}


beggars can't be choosers. Finally, $\mu_{1}$ Soo and $\mu_{2}$ Sco appear in the photometer field together and this observation must be considered the average of the two stars. Since they are similar in spectral type and colour, the observation should still have weight.

The ultraviolet colours listed are absolute. That is, they represent true ratios of the $2200 \AA$ and $2600 \AA$ fluxes. It is fortuitous that early $\mathrm{A}$ stars have roughly equal fluxes at these two wavelengths, making the zero point of the ultraviolet colour scale nearly correct. The curve drawn in

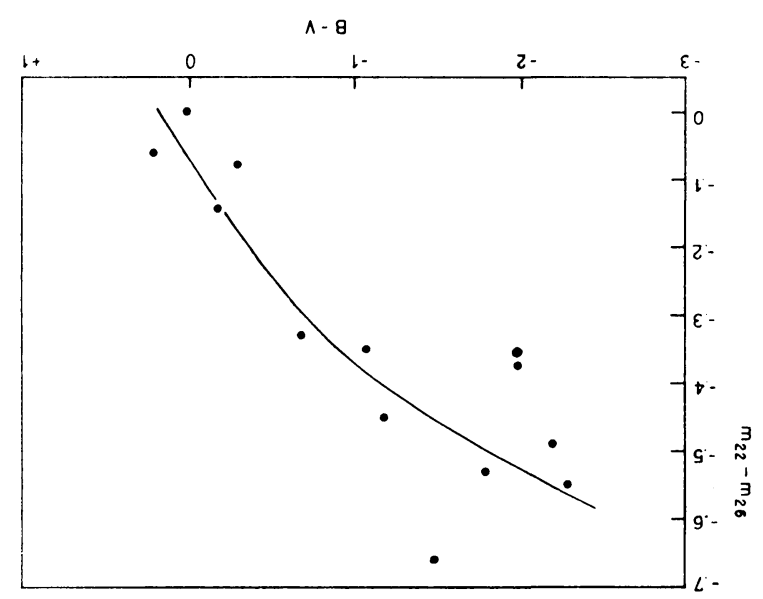

Fra. 3. - Colour-Colour diagram for well observed stars of luminosity classes III, IV, and $\mathrm{V}$ having $\mathrm{E} \leqslant .02$.
Figure 3 is arbitrary. However, all but three of the stars are within one-tenth magnitude of that curve, and those three are within two-tenths. This degree of correlation appears to be comparable to the accuracy of the data. Such a correlation is very encouraging. It means, for instance, that it should be possible to derive information on interstellar scattering in the ultraviolet, and, of course, to derive a temperature scale for the B stars based on the observed ultraviolet spectral gradient. This phase of the work is just starting, but STECHER will describe some of the first attempts in the following paper in connection with the spectrophotometric data. There again, the matter of calibration is all important. Fortunately there are seven stars in common between the broadband and the spectrophotometric data, so that spectrophotometric fluxes at 2200 and $2600 \AA$ can be compared with fluxes from the filter photometry. The seven stars are $\varepsilon$ and $\eta \mathrm{UMa}, \gamma$ Cas, $\beta$ Cep, $\tau$, $\zeta$ and $\pi$ Sco. The two sets of data, which are obtained by quite different techniques, agree within $20 \%$ for all seven of these stars, and for four out of the seven there is agreement within $10 \%$. In other words, we are now at the stage where the observations are sufficiently reliable to be used with confidence in deriving information on stellar atmospheres and interstellar scattering.

Manuscrit reçu le 27 septembre 1964. 